\title{
Electromagnetic form factors of charged and neutral kaons in an extended vector-meson-dominance model
}

\author{
S.A. Ivashyn $1, *$ and A.Yu. Korchin ${ }^{2},+$ \\ ${ }^{1}$ Kharkov National University, Kharkov 6107r, Ukraine \\ ${ }^{2}$ NSC "Kharkov Institute of Physics and Technology", Kharkov 61108, Ukraine

\begin{abstract}
A model is developed for electromagnetic form factors of the charged and neutral
\end{abstract} \\ $K$-mesons. The formalism is based on ChPT Lagrangians with vector mesons. The form \\ factors, calculated without fitting parameters, are in a good agreement with experiment for \\ space-like and time-like photon momenta. Contribution of the two-kaon channels to the \\ muon anomalous magnetic moment $a_{\mu}$ is calculated. \\ PACS numbers: 12.39.Fe, 12.40.Vv, 13.40.Gp, 13.66.Bc
}

\section{INTRODUCTION}

Hadronic contribution to the vacuum polarization plays an important role in the test of the Standard Model at the electroweak precision level. It is the main source of the theoretical uncertainties in value of the muon anomalous magnetic moment $a_{\mu}=\left(g_{\mu}-2\right) / 2$. The dominant piece of the hadronic contribution is the $\pi^{+} \pi^{-}$channel, which is expressed in terms of the pion electromagnetic form factor $(\mathrm{FF}) F_{\pi}(s)$. This $\mathrm{FF}$ has been studied during the last years both experimentally 1] and theoretically (e.g., in hadronic models [2, 3]). The two-pion channel at low energies accounts for about $70 \%$ of the leading order $(\mathrm{LO})$ total hadronic contribution to $a_{\mu}$, at the same time the other hadronic channels are also important. High precision measurements of various hadronic channels were performed by CMD-2 and SND in Novosibirsk, and of $\pi^{+} \pi^{-}$channel by KLOE in Frascati.

In this paper we concentrate on the electromagnetic (EM) FF's of the kaons, the charged ones $K^{+}, K^{-}$, and the neutral ones $K^{0}, \bar{K}^{0}$, mainly in the time-like region of the momentum transfer. Experimental information on these FF's comes from measurement of the total cross section of the electron-positron annihilation $e^{+} e^{-} \rightarrow K^{+} K^{-}$:

$$
\sigma\left(e^{+} e^{-} \rightarrow K^{+} K^{-}\right)=\frac{\pi \alpha^{2}}{3 s}\left(1-\frac{4 m_{K}^{2}}{s}\right)^{3 / 2}\left|F_{K^{+}}(s)\right|^{2}
$$

\footnotetext{
*Electronic address: ivashin.s@rambler.ru

${ }^{\dagger}$ Electronic address: korchin@kipt.kharkov.ua
} 
and similarly for $e^{+} e^{-} \rightarrow K_{S} K_{L}$. In (1) $s$ is the squared total energy in center of mass (CM) frame, $\alpha$ is the fine-structure constant, $m_{K}$ is the $K$-meson mass (the electron mass in (11) is neglected).

New accurate data on the neutral kaon FF's have recently been obtained in Novosibirsk (CMD-2 collaboration [4] and SND collaboration [5]), and results for the charged kaon are being analyzed. The kaon FF's are conventionally studied in terms of the intermediate $J^{P}=1^{-}$mesons: $\rho(770)$, $\omega(782)$ and $\phi(1020)$. It turns out that at energies above $\sqrt{s} \approx 1 \mathrm{GeV}$ vector resonances $\rho^{\prime}=\rho(1450)$, $\omega^{\prime}=\omega(1420)$ and $\phi^{\prime}=\phi(1680)$, which are the low-lying radial excitations of the $\rho(770), \omega(782)$ and $\phi(1020)$ respectively, also begin to play an important role. The properties of the latter resonances are not well known. Recently a model has been worked out [6] which accounts for main vectormeson contributions. The parameters within approach [6] have been fitted to existing data on pion and kaon FF's.

In the present paper we develop a model for the charged and neutral kaon EM FF's in both the time-like region (at $\sqrt{s}=1-2 \mathrm{GeV}$ ) and space-like region, $s \leq 0$. It is based on ChPT with vector mesons [7, 8]. On the tree-diagrams level the process $e^{+} e^{-} \rightarrow K^{+} K^{-}\left(\right.$or $\left.e^{+} e^{-} \rightarrow K^{0} \bar{K}^{0}\right)$ is described via intermediate $\rho(770), \omega(782), \phi(1020)$ states (and possibly including other $J^{P}=1^{-}$ resonances), the couplings of which to the photon, $f_{V}$, are related by the flavor $S U(3)$ symmetry. The couplings to the kaons, $g_{V K^{+} K^{-}}$and $g_{V K^{0} \bar{K}^{0}}$, also obey $S U(3)$ symmetry relations. It is also possible to determine some of these couplings from decay widths, and this way is preferable as the $S U(3)$ symmetry is not exact. In this model the photon vector-meson vertices by construction depend on the photon momentum $q$ and explicitly guarantee gauge invariance of the theory. These properties are important because they automatically give the correct normalization of FF's, $F_{K^{+}}\left(q^{2}=0\right)=1$ and $F_{K^{0}}\left(q^{2}=0\right)=0$, naturally account for the energy dependence of the EM vertices and the vertices vanish in the case of real-photon vector meson transition.

In the model we include certain loop corrections beyond the tree level. In particular, there are self-energy polarization operators that modify vector-meson propagators and loop corrections that lead to "dressing" of the photon-meson vertices. For construction of vertices in the odd-intrinsicparity sector, when necessary, we apply the chiral anomalous Lagrangian of Wess-Zumino-Witten (WZW) [9, 10] for $\gamma \Phi \Phi \Phi$ interaction and chiral Lagrangians [11, 12] for $V \gamma \Phi, V \Phi \Phi$ and $V \Phi \Phi \Phi$ interactions. As an option we also use method of Ref. [13] to constrain parameters of the interaction of the vector mesons. Parameters of the model are fixed from decay widths of the vector mesons, and the kaon FF's are calculated without fitting the parameters.

The plan of the paper is as follows. Sect. II contains formalism needed to describe the photon, vector-meson and pseudoscalar-meson interactions. The formalism is based on ChPT Lagrangians 
with vector mesons in the even and odd intrinsic-parity sectors. In sect. III this formalism is applied to calculation of the kaon form factors. The model is extended by using the "dressed" propagators and vertices, and adding the higher resonances. Results of calculations and discussion are presented in sect. IV] The $K \bar{K}$-channel contribution to muon anomalous magnetic moment $a_{\mu}$ is also calculated. In sect. $\nabla$ we draw conclusions. Appendix A contains ChPT Lagrangian with vector mesons.

\section{FORMALISM}

\section{A. ChPT Lagrangian}

In the even-intrinsic-parity sector we apply ChPT Lagrangian of Ecker et al. 7, 8] (see Appendix A for details) which is an appropriate model in the energy region of interest. The antisymmetric tensor formulation for the spin-1 fields is used as it directly fulfills the high-energy constraints of QCD (the model I of [8]). The other possibility, vector formulation, is shown [8] to be equivalent to the tensor formulation to order $\mathcal{O}\left(p^{4}\right)$ only if additional local terms of order $\mathcal{O}\left(p^{4}\right)$ are present in Lagrangian.

Expansion in powers of momenta describes standard EM interaction of the charged pseudoscalar fields $\Phi$

$$
\begin{gathered}
\mathcal{L}_{\gamma \Phi \Phi}^{(1)}=\imath e B_{\mu} \operatorname{Tr}\left(Q\left[\partial_{\mu} \Phi, \Phi\right]\right)=-\imath e B_{\mu}\left(\pi^{+} \hat{\partial}^{\mu} \pi^{-}+K^{+} \hat{\partial}^{\mu} K^{-}\right) \\
\mathcal{L}_{\gamma \gamma \Phi \Phi}^{(2)}=-\frac{e^{2}}{2} B^{\mu} B_{\mu} \operatorname{Tr}\left([\Phi, Q]^{2}\right)=e^{2} B^{\mu} B_{\mu}\left(\pi^{+} \pi^{-}+K^{+} K^{-}\right)
\end{gathered}
$$

where notation $\pi^{+} \hat{\partial}^{\mu} \pi^{-} \equiv \pi^{+}\left(\partial^{\mu} \pi^{-}\right)-\left(\partial^{\mu} \pi^{+}\right) \pi^{-}$, etc. has been introduced and $e=\sqrt{4 \pi \alpha} \approx 0.303$ (see Appendix A for definition of $\Phi$ and quark charge matrix $Q$ ). One also obtains direct photon vector-meson coupling

$$
\mathcal{L}_{\gamma V}=e \frac{F_{V}}{\sqrt{2}} F^{\mu \nu} \operatorname{Tr}\left(V_{\mu \nu} Q\right)=e F_{V} F^{\mu \nu}\left(\frac{1}{2} \rho_{\mu \nu}^{0}+\frac{1}{6} \omega_{\mu \nu}-\frac{1}{3 \sqrt{2}} \phi_{\mu \nu}\right) .
$$

This Lagrangian is explicitly gauge invariant and leads to momentum-dependent vertices for the $\langle\gamma(\mu) \mid V(\nu \rho)\rangle$ transition, which are proportional to $e F_{V}\left(q^{\nu} g^{\rho \mu}-q^{\rho} g^{\mu \nu}\right)$, where $\mu, \nu, \rho=0, \ldots, 3$ are the Lorentz indices. 


\begin{tabular}{|c|c|c|c|}
\hline & $\rho^{0}$ & $\omega$ & $\phi$ \\
\hline$S U(3): f_{V}$ & $f$ & $3 f$ & $-\frac{3}{\sqrt{2}} f$ \\
\hline exp.: $\Gamma\left(V \rightarrow e^{+} e^{-}\right), \mathrm{keV}$ & $7.02 \pm 0.11$ & $0.60 \pm 0.02$ & $1.27 \pm 0.04$ \\
$f_{V}$ & $4.966 \pm 0.038$ & $17.06 \pm 0.29$ & $-13.38 \pm 0.21$ \\
\hline exp.: $\Gamma\left(V \rightarrow \mu^{+} \mu^{-}\right), \mathrm{keV}$ & $6.66 \pm 0.2$ & $0.76 \pm 0.26$ & $1.21 \pm 0.08$ \\
$f_{V}$ & $5.09 \pm 0.08$ & $15.15 \pm 2.8$ & $-13.71 \pm 0.45$ \\
\hline
\end{tabular}

Table I: Values of the EM coupling constants for $V=\rho^{0}, \omega, \phi$.

The interaction of vector mesons with pseudoscalars is given by

$$
\begin{aligned}
\mathcal{L}_{V \Phi \Phi}= & \imath \frac{\sqrt{2} G_{V}}{F_{\pi}^{2}} \operatorname{Tr}\left(V_{\mu \nu} \partial^{\mu} \Phi \partial^{\nu} \Phi\right) \\
= & \imath \frac{G_{V}}{F_{\pi}^{2}}\left[\rho_{\mu \nu}^{0}\left(2 \partial^{\mu} \pi^{+} \partial^{\nu} \pi^{-}+\partial^{\mu} K^{+} \partial^{\nu} K^{-}-\partial^{\mu} K^{0} \partial^{\nu} \bar{K}^{0}\right)\right. \\
& \left.+\omega_{\mu \nu}\left(\partial^{\mu} K^{+} \partial^{\nu} K^{-}+\partial^{\mu} K^{0} \partial^{\nu} \bar{K}^{0}\right)+\phi_{\mu \nu}\left(-\sqrt{2} \partial^{\mu} K^{+} \partial^{\nu} K^{-}-\sqrt{2} \partial^{\mu} K^{0} \partial^{\nu} \bar{K}^{0}\right)\right] .
\end{aligned}
$$

In deriving (5) the antisymmetry of the vector fields, $V_{\mu \nu}=-V_{\nu \mu}$, has been used. The pion weakdecay constant is $F_{\pi}=92.4 \mathrm{MeV}$.

The coupling constants $F_{V}$ and $G_{V}$ can be found from experimental widths of decays $\Gamma(\rho \rightarrow$ $\left.e^{+} e^{-}\right)$and $\Gamma(\rho \rightarrow \pi \pi)$, respectively. It will be further convenient to use other constants, $g$ and $f$, related to $F_{V}$ and $G_{V}$. Using the data from [14] we obtain

$$
\begin{gathered}
F_{V}=156.35 \mathrm{MeV}, \quad G_{V}=65.65 \mathrm{MeV}, \\
f \equiv \frac{m_{\rho}}{F_{V}}=4.966, \quad g \equiv \frac{G_{V} m_{\rho}}{F_{\pi}^{2}}=5.965 .
\end{gathered}
$$

In (44) and (5) the exact $S U(3)$ symmetry is supposed. To have a more flexible model we introduce for each vector meson independent EM couplings $f_{V}=\left(f_{\rho}, f_{\omega}, f_{\phi}\right)$ and strong couplings $g_{\rho \pi \pi}, g_{\rho K^{+} K^{-}}, g_{\rho K^{0} \bar{K}^{0}}, g_{\omega K^{+} K^{-}}, g_{\omega K^{0} \bar{K}^{0}}$ and $g_{\phi K^{+} K^{-}}, g_{\phi K^{0} \bar{K}^{0}}$. The $S U(3)$ relations for these constants are shown in Tables \ and II. The couplings $f_{V}$ can be directly fixed from the decay

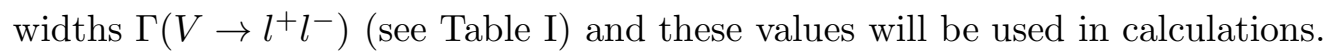

The odd-intrinsic-parity interactions are discussed in the following subsections. 


\begin{tabular}{|c|c|c|c|}
\hline & $\pi^{+} \pi^{-}$ & $K^{+} K^{-}$ & $K^{0} \bar{K}^{0}$ \\
\hline$\rho^{0}$ & $g$ & $\frac{1}{2} g$ & $-\frac{1}{2} g$ \\
$\omega$ & - & $\frac{1}{2} g$ & $\frac{1}{2} g$ \\
$\phi$ & - & $-\frac{1}{\sqrt{2}} g$ & $-\frac{1}{\sqrt{2}} g$ \\
\hline
\end{tabular}

Table II: $S U(3)$ values of the vector-meson coupling to two pseudoscalar mesons.

\section{B. Photon - pseudoscalar interaction}

The WZW Lagrangian [9, 10] involving interaction of pseudoscalar mesons with external EM field reads

$$
\begin{aligned}
\mathcal{L}_{W Z W}= & \mathcal{L}_{W Z W}^{(1)}+\mathcal{L}_{W Z W}^{(2)}, \\
\mathcal{L}_{W Z W}^{(1)}= & -\frac{e N_{c}}{48 \pi^{2}} \epsilon^{\mu \nu \alpha \beta} B_{\mu} \operatorname{Tr}\left(Q\left[\left(\partial_{\nu} U\right)\left(\partial_{\alpha} U^{+}\right)\left(\partial_{\beta} U\right) U^{+}-\left(\partial_{\nu} U^{+}\right)\left(\partial_{\alpha} U\right)\left(\partial_{\beta} U^{+}\right) U\right]\right), \\
\mathcal{L}_{W Z W}^{(2)}= & \frac{e^{2} N_{c}}{24 \pi^{2}} \epsilon^{\mu \nu \alpha \beta}\left(\partial_{\mu} B_{\nu}\right) B_{\alpha} \\
& \times \operatorname{Tr}\left(Q^{2}\left(\partial_{\beta} U\right) U^{+}+Q^{2} U^{+}\left(\partial_{\beta} U\right)-\frac{1}{2} Q U Q\left(\partial_{\beta} U^{+}\right)+\frac{1}{2} Q U^{+} Q\left(\partial_{\beta} U\right)\right),
\end{aligned}
$$

where $N_{c}=3$ is the number of quark colors, $\epsilon_{0123}=-\epsilon^{0123}=1$ and $U=\exp \left(i \sqrt{2} \Phi / F_{\pi}\right)$.

We keep in (7) the lowest-order EM interaction with three and one pseudoscalar mesons:

$$
\begin{aligned}
\mathcal{L}_{\gamma \Phi \Phi \Phi} & =-\frac{\imath \sqrt{2} e N_{c}}{12 \pi^{2} F_{\pi}^{3}} \epsilon^{\mu \nu \alpha \beta} B_{\mu} \operatorname{Tr}\left(Q \partial_{\nu} \Phi \partial_{\alpha} \Phi \partial_{\beta} \Phi\right) \\
& =\frac{\imath e N_{c}}{12 \pi^{2} F_{\pi}^{3}} \epsilon^{\mu \nu \alpha \beta} B_{\mu}\left(\partial_{\nu} \pi^{-} \partial_{\alpha} \pi^{+} \partial_{\beta} \pi^{0}+2 \partial_{\nu} \pi^{0} \partial_{\alpha} K^{-} \partial_{\beta} K^{+}+\partial_{\nu} \pi^{0} \partial_{\alpha} \bar{K}^{0} \partial_{\beta} K^{0}\right) \\
\mathcal{L}_{\gamma \gamma \Phi} & =-\frac{\sqrt{2} e^{2} N_{c}}{8 \pi^{2} F_{\pi}} \epsilon^{\mu \nu \alpha \beta} \partial_{\mu} B_{\nu} \partial_{\alpha} B_{\beta} \operatorname{Tr}\left(Q^{2} \Phi\right) .
\end{aligned}
$$

The latter, in particular, describes anomalous $\pi^{0} \gamma \gamma$ interaction

$$
\mathcal{L}_{\gamma \gamma \pi^{0}}=-\frac{e^{2} N_{c}}{24 \pi^{2} F_{\pi}} \epsilon^{\mu \nu \alpha \beta} \partial_{\mu} B_{\nu} \partial_{\alpha} B_{\beta} \pi^{0}
$$

\section{Vector-pseudoscalar-photon interactions}

For the odd-intrinsic-parity interactions of vector mesons we use chiral Lagrangians in vector formulation for spin-1 fields [11, 12]. As shown in [11] the use of vector fields for $1^{-}$resonances ensures correct behavior of Green functions to order $\mathcal{O}\left(p^{6}\right)$. In the alternative tensor formulation, however, local terms of $\mathcal{O}\left(p^{6}\right)$ would be explicitly needed to fulfill high-energy constraints of QCD. 
Expanding the corresponding Lagrangian (see Appendix A) we find

$$
\begin{aligned}
\mathcal{L}_{V V \Phi} & =-\frac{\sqrt{2} \sigma_{V}}{F_{\pi}} \epsilon^{\mu \nu \alpha \beta} \operatorname{Tr}\left(\partial_{\mu} V_{\nu}\left\{\Phi, \partial_{\alpha} V_{\beta}\right\}\right), \\
\mathcal{L}_{V \gamma \Phi} & =-\frac{4 \sqrt{2} e h_{V}}{F_{\pi}} \epsilon^{\mu \nu \alpha \beta} \partial_{\mu} B_{\nu} \operatorname{Tr}\left(V_{\alpha}\left\{\partial_{\beta} \Phi, Q\right\}\right) .
\end{aligned}
$$

These terms induce the interactions

$$
\begin{aligned}
\mathcal{L}_{\omega \rho \pi} & =-\frac{4 \sigma_{V}}{F_{\pi}} \epsilon^{\mu \nu \alpha \beta} \partial_{\mu} \omega_{\nu}\left(\vec{\pi} \partial_{\alpha} \vec{\rho}_{\beta}\right) \\
\mathcal{L}_{V \gamma \pi^{0}} & =-\frac{4 \sqrt{2} e h_{V}}{3 F_{\pi}} \epsilon^{\mu \nu \alpha \beta} \partial_{\mu} B_{\nu}\left(\rho_{\alpha}^{0}+3 \omega_{\alpha}+3 \epsilon_{\omega \phi} \phi_{\alpha}\right) \partial_{\beta} \pi^{0} .
\end{aligned}
$$

The last term in (14) represents the OZI-forbidden process, and $\epsilon_{\omega \phi}=0.058$ [2] is the $\omega$ - $\phi$ mixing parameter. The $V \Phi \Phi \Phi$ interaction is

$$
\begin{aligned}
\mathcal{L}_{V \Phi \Phi \Phi}= & -\frac{2 \imath \sqrt{2} \theta_{V}}{F_{\pi}^{3}} \epsilon^{\mu \nu \alpha \beta} \operatorname{Tr}\left(V_{\mu} \partial_{\nu} \Phi \partial_{\alpha} \Phi \partial_{\beta} \Phi\right) \\
= & -\frac{2 \imath \sqrt{2} \theta_{V}}{F_{\pi}^{3}} \epsilon^{\mu \nu \alpha \beta}\left(\rho_{\mu}^{0} \partial_{\nu} \pi^{0} \partial_{\alpha} K^{+} \partial_{\beta} K^{-}+\rho_{\mu}^{0} \partial_{\nu} \pi^{0} \partial_{\alpha} K^{0} \partial_{\beta} \bar{K}^{0}+3 \omega_{\mu} \partial_{\nu} \pi^{0} \partial_{\alpha} \pi^{+} \partial_{\beta} \pi^{-}\right. \\
& +\omega_{\mu} \partial_{\nu} \pi^{0} \partial_{\alpha} K^{+} \partial_{\beta} K^{-}-\omega_{\mu} \partial_{\nu} \pi^{0} \partial_{\alpha} K^{0} \partial_{\beta} \bar{K}^{0}+\sqrt{2} \omega_{\mu} \partial_{\nu} \pi^{+} \partial_{\alpha} K^{0} \partial_{\beta} K^{-} \\
& +\sqrt{2} \omega_{\mu} \partial_{\nu} \pi^{-} \partial_{\alpha} K^{+} \partial_{\beta} \bar{K}^{0}+\frac{1}{\sqrt{2}} \phi_{\mu} \partial_{\nu} \pi^{0} \partial_{\alpha} K^{+} \partial_{\beta} K^{-}+\frac{1}{\sqrt{2}} \phi_{\mu} \partial_{\nu} \pi^{0} \partial_{\alpha} \bar{K}^{0} \partial_{\beta} K^{0} \\
& \left.+\phi_{\mu} \partial_{\nu} \pi^{+} \partial_{\alpha} K^{0} \partial_{\beta} K^{-}+\phi_{\mu} \partial_{\nu} \pi^{-} \partial_{\alpha} K^{+} \partial_{\beta} \bar{K}^{0}\right) .
\end{aligned}
$$

The parameter $h_{V}$ is fixed to the value $h_{V}=0.039$ from the width of the $\omega \rightarrow \gamma \pi^{0}$ decay. To obtain $\theta_{V}$ and $\sigma_{V}$ we use experimental values [14] for the three-pion decay widths of $\omega$ and $\phi$ mesons [15]. It is usually supposed that the $\phi$-meson decays into the three pions via the $\omega-\phi$ mixing (for other options see [16, 17, 18]), and that the amplitudes for the direct decay, $\phi \rightarrow \pi \pi \pi$, and decay through the intermediate state, $\phi \rightarrow \rho \pi \rightarrow \pi \pi \pi$, sum up incoherently. According to [19, 20, 21] the direct $\omega \rightarrow \pi \pi \pi$ (and $\phi \rightarrow \pi \pi \pi$ ) decay is suppressed with respect to the process $\omega \rightarrow \rho \pi \rightarrow \pi \pi \pi$ (and $\phi \rightarrow \rho \pi \rightarrow \pi \pi \pi$ ). In view of these constraints we obtain $\sigma_{V}=0.33$ and $\theta_{V}=0.0011$.

There is an interesting approach [13] which generalizes the WZW anomaly term [9, 10] for the case of vector and axial-vector mesons. The authors of [13] applied Bardeen's form of anomaly to keep the vector current anomaly free and have anomaly for the axial-vector current. The $V \Phi \Phi \Phi$ and $V V \Phi$ pieces of Lagrangian in this approach have the form

$$
\begin{aligned}
\mathcal{L}_{V \Phi \Phi \Phi} & =-\frac{\imath g}{4 \pi^{2} F_{\pi}^{3}} \epsilon^{\mu \nu \alpha \beta} \operatorname{Tr}\left(V_{\mu} \partial_{\nu} \Phi \partial_{\alpha} \Phi \partial_{\beta} \Phi\right) \\
\mathcal{L}_{V V \Phi} & =-\frac{3 g^{2}}{16 \sqrt{2} \pi^{2} F_{\pi}} \epsilon^{\mu \nu \alpha \beta} \operatorname{Tr}\left(\partial_{\mu} V_{\nu}\left\{\Phi, \partial_{\alpha} V_{\beta}\right\}\right) .
\end{aligned}
$$




\begin{tabular}{|c|c|c|c|}
\hline Parameters & $h_{V}$ & $\theta_{V}$ & $\sigma_{V}$ \\
\hline "ideal" values & $\frac{3 g}{32 \sqrt{2} \pi^{2}}=0.040$ & $\frac{g}{8 \sqrt{2} \pi^{2}}=0.054$ & $\frac{3 g^{2}}{32 \pi^{2}}=0.34$ \\
\hline fixed from experiment & 0.039 & 0.0011 & 0.33 \\
\hline values in model [12] for $g_{A}=1$ & $\frac{N_{c} f^{\prime}}{32 \sqrt{2} \pi^{2}}=0.040$ & $\frac{N_{c} f^{\prime}}{24 \sqrt{2} \pi^{2}}=0.053$ & $\frac{N_{c} f^{\prime 2}}{32 \pi^{2}}=0.33$ \\
\hline
\end{tabular}

Table III: Values of parameters in Lagrangians (11), (12) and (15). $g=5.965$ is fixed from $\rho \rightarrow \pi \pi$ decay, and $f^{\prime}=1 / 0.17 \approx 5.9[12]$.

The EM field can be included, based on $U(1)$ gauge invariance and vector-meson dominance, by the substitution

$$
V_{\mu} \rightarrow V_{\mu}+\frac{\sqrt{2} e}{g} Q B_{\mu}
$$

in the covariant derivative [13] acting on $U$. By substituting (18) in (17) one obtains $V \gamma \Phi$ interaction

$$
\mathcal{L}_{V \gamma \Phi}=-\frac{3 e g}{8 \pi^{2} F_{\pi}} \epsilon^{\mu \nu \alpha \beta} \partial_{\mu} B_{\nu} \operatorname{Tr}\left(V_{\alpha}\left\{\partial_{\beta} \Phi, Q\right\}\right)
$$

and $\gamma \gamma \Phi$ Lagrangian (9). Further, substitution of (18) in (16) leads to $\gamma \Phi \Phi \Phi$ interaction (8).

Eqs.(16), (17) and (19) can be used to estimate parameters $h_{V}, \theta_{V}$ and $\sigma_{V}$ in (11), (12) and (15) (we call the corresponding values "ideal" ones). These values are shown in the 2nd line of Table III and the values determined from experiment are listed in the 3rd line. It is seen that the parameters $h_{V}$ and $\sigma_{V}$ agree well, while the experiment favors very small value of the constant $\theta_{V}$ for the $V \Phi \Phi \Phi$ vertex.

In the last line of Table III we present results for these couplings in an extended Nambu-JonaLasinio model of QCD [12]. For a particular value of axial constant $g_{A}=1$, and $f^{\prime} \approx 5.9$ from [12], the obtained couplings agree with the "ideal" values.

\section{KAON ELECTROMAGNETIC FORM FACTORS}

The kaon EM FF's can be defined in terms of the quark EM current (see, e.g., [22])

$$
\begin{aligned}
j_{e m}^{\mu}(x) & =\frac{2}{3} \bar{u}(x) \gamma^{\mu} u(x)-\frac{1}{3} \bar{d}(x) \gamma^{\mu} d(x)-\frac{1}{3} \bar{s}(x) \gamma^{\mu} s(x) \\
& =V_{3}^{\mu}(x)+\frac{1}{\sqrt{3}} V_{8}^{\mu}(x),
\end{aligned}
$$

where the isovector and isoscalar components are respectively

$$
\begin{aligned}
V_{3}^{\mu}(x) & =\bar{q} \gamma^{\mu} \frac{\lambda_{3}}{2} q=\frac{1}{2}\left(\bar{u} \gamma^{\mu} u-\bar{d} \gamma^{\mu} d\right) \\
\frac{1}{\sqrt{3}} V_{8}^{\mu}(x) & =\frac{1}{\sqrt{3}} \bar{q} \gamma^{\mu} \frac{\lambda_{8}}{2} q=\frac{1}{6}\left(\bar{u} \gamma^{\mu} u+\bar{d} \gamma^{\mu} d\right)-\frac{1}{3} \bar{s} \gamma^{\mu} s
\end{aligned}
$$


Then the FF's are defined through the matrix elements

$$
\begin{aligned}
\left\langle K^{+}\left(p_{1}\right) K^{-}\left(p_{2}\right)\left|j_{e m}^{\mu}(0)\right| 0\right\rangle & \equiv\left(p_{1}-p_{2}\right)^{\mu} F_{K^{+}}\left(q^{2}\right), \\
\left\langle K^{0}\left(p_{1}\right) \bar{K}^{0}\left(p_{2}\right)\left|j_{e m}^{\mu}(0)\right| 0\right\rangle & \equiv\left(p_{1}-p_{2}\right)^{\mu} F_{K^{0}}\left(q^{2}\right),
\end{aligned}
$$

where $p_{1}, p_{2}$ are the kaon momenta, $q=p_{1}+p_{2}$ and $q^{2} \equiv s$. These FF's, being defined in the timelike region $s \geq 4 m_{K}^{2}$, due to the analyticity describe also the space-like region $s<0$ corresponding to elastic electron scattering on the kaon.

The direct $\gamma V$ coupling in (4) leads to the following form for FF's:

$$
\begin{gathered}
F_{K^{+}}(s)=1-\sum_{V=\rho, \omega, \phi} \frac{g_{V K^{+} K^{-}}}{f_{V}(s)} A_{V}(s), \quad F_{K^{0}}(s)=-\sum_{V=\rho, \omega, \phi} \frac{g_{V K^{0} \bar{K}^{0}}}{f_{V}(s)} A_{V}(s), \\
A_{V}(s) \equiv \frac{s}{s-m_{V}^{2}-\Pi_{V}(s)},
\end{gathered}
$$

where $\Pi_{V}(s)$ is the self-energy operator for the vector meson $V=\rho, \omega, \phi$.

It is seen that due to gauge invariance of the photon vector-meson interaction the coupling is proportional to photon invariant mass $s$ and vanishes for real photons. This automatically leads to the correct normalization conditions

$$
F_{K^{+}}(0)=1, \quad F_{K^{0}}(0)=0 .
$$

Thus there is no need to impose constraints on coefficients in (23) in order to obey (25). An additional energy dependence of the couplings $f_{V}(s)$ arises due to higher-order corrections, as described in subsect. IIIB.

Using for the strong couplings the $S U(3)$ relations in Table II we can write

$$
\begin{aligned}
& F_{K^{+}}(s)=1-\frac{g}{2 f_{\rho}(s)} A_{\rho^{0}}(s)-\frac{g}{2 f_{\omega}(s)} A_{\omega}(s)+\frac{g}{\sqrt{2} f_{\phi}(s)} A_{\phi}(s), \\
& F_{K^{0}}(s)=\frac{g}{2 f_{\rho}(s)} A_{\rho^{0}}(s)-\frac{g}{2 f_{\omega}(s)} A_{\omega}(s)+\frac{g}{\sqrt{2} f_{\phi}(s)} A_{\phi}(s) .
\end{aligned}
$$

\section{A. Self-energy operators}

In general the dressed propagator of vector particles includes the self-energy operators $\Pi_{V}(s)$. The latter should account for all intermediate states, such as $\pi^{+} \pi^{-}, \omega \pi^{0}, K \bar{K}, \omega \pi^{0} \rightarrow \pi^{0} K^{+} K^{-}$ for $\rho$ meson, $K \bar{K}, \rho \pi, \pi^{0} \pi^{+} \pi^{-}, \rho \pi \rightarrow \pi^{0} \pi^{+} \pi^{-}, \rho \pi \rightarrow \pi^{0} K \bar{K}$ for $\omega$ meson, $K \bar{K}, \rho \pi, \pi^{0} K \bar{K}$, $\rho \pi \rightarrow \pi^{0} K \bar{K}, \pi^{0} \pi^{+} \pi^{-}, \rho \pi \rightarrow \pi^{0} \pi^{+} \pi^{-}$for $\phi$ meson (including those with $\omega-\phi$ mixing), and possibly others. As our calculations indicates, in the region of interest the most important contributions 
to $\Pi_{V}(s)$ consist of the diagrams shown in Fig. 1. These diagrams are the dominant ones in the energy interval $\sqrt{s}$ about few GeV's, they contribute to the self-energy for $\rho, \omega$ and $\phi$ mesons about $86 \%, 95 \%$ and $95 \%$ respectively. We take into consideration that there is experimental evidence [19, 20, 21] of a relatively small direct couplings $\omega, \phi \rightarrow \pi \pi \pi$.
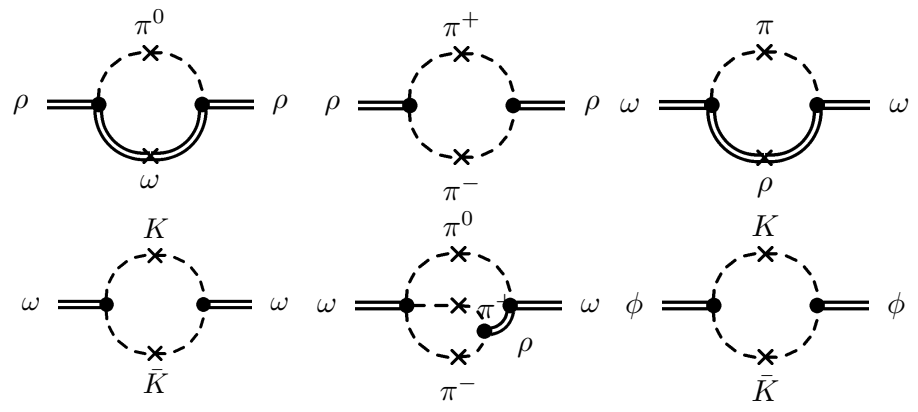

Figure 1: Loops included in self-energy of vector mesons. Crosses indicate imaginary part of the diagrams.

These diagrams can be collected in the self-energy operators as follows

$$
\begin{aligned}
& \Pi_{\rho}=\Pi_{\rho\left(\pi^{0} \omega\right) \rho}+\Pi_{\rho(\pi \pi) \rho} \\
& \Pi_{\omega}=\Pi_{\omega\left(\pi^{0} \rho\right) \omega}+\Pi_{\omega(K K) \omega}+2 \Pi_{\omega(3 \pi, \pi \rho) \omega} \\
& \Pi_{\phi}=\Pi_{\phi(K K) \phi}
\end{aligned}
$$

where the subscript notation is explained in Fig. 1,

In the following we include only the imaginary parts of the loop contributions. These will be the dominant contributions giving rise to the energy-dependent widths

$$
\Gamma_{V}(s)=-m_{V}^{-1} \operatorname{Im} \Pi_{V}(s)
$$

The real part of the self-energy, $\operatorname{Re} \Pi_{V}(s)$, usually can be taken into account by an appropriate renormalization of the coupling constants and masses. An approximation, consisting in neglecting the real part of the loop contributions compared to the imaginary part, is often used in scattering theory and is known as $K$-matrix approach. For an example of successful application of the coupled-channel $K$-matrix approach to reactions on the nucleon see Ref. [23].

Applying the Cutkosky rules ([22], Ch.6.3) to the diagrams shown in Fig. 11we find the imaginary 
parts:

$$
\begin{aligned}
& \operatorname{Im}_{\rho\left(\pi^{0} \omega\right) \rho}(s)=\frac{-\sqrt{s}}{12 \pi} \frac{16 \sigma_{V}^{2}}{F_{\pi}^{2}}\left(\frac{\left(s+m_{\pi}^{2}-m_{\omega}^{2}\right)^{2}}{4 s}-m_{\pi}^{2}\right)^{3 / 2} \theta\left(s-\left(m_{\pi}+m_{\omega}\right)^{2}\right), \\
& \operatorname{Im}_{\rho(\pi \pi) \rho}(s)=\frac{-g^{2}}{48 \pi \sqrt{s}}\left(s-4 m_{\pi}^{2}\right)^{3 / 2} \theta\left(s-4 m_{\pi}^{2}\right), \\
& \operatorname{Im}_{\omega\left(\pi^{0} \rho\right) \omega}(s)=\frac{-\sqrt{s}}{12 \pi} \frac{16 \sigma_{V}^{2}}{F_{\pi}^{2}}\left(\frac{\left(s+m_{\pi}^{2}-m_{\rho}^{2}\right)^{2}}{4 s}-m_{\pi}^{2}\right)^{3 / 2} \theta\left(s-\left(m_{\pi}+m_{\rho}\right)^{2}\right), \\
& \operatorname{Im} \Pi_{\omega(K K) \omega}(s)=\frac{-\frac{1}{2} g^{2}}{48 \pi \sqrt{s}}\left(s-4 m_{K}^{2}\right)^{3 / 2} \theta\left(s-4 m_{K}^{2}\right), \\
& \operatorname{Im} \Pi_{\phi(K K) \phi}(s)=\frac{-g^{2}}{48 \pi \sqrt{s}}\left(s-4 m_{K}^{2}\right)^{3 / 2} \theta\left(s-4 m_{K}^{2}\right), \\
& \operatorname{Im} \Pi_{\omega(3 \pi, \pi \rho) \omega}(s)=\frac{s}{2^{8} \pi^{3}} \int d E_{+} d E_{-} 4\left(\left(\vec{p}_{-} \vec{p}_{+}\right)^{2}-\vec{p}_{-}^{2} \vec{p}_{+}{ }^{2}\right) \\
& \times\left[-\frac{g}{3} \frac{24 \sqrt{2} h_{V} \sigma_{V}}{2 F_{\pi}^{4}} \operatorname{Im}_{p_{\rho}^{2}-m_{\rho}^{2}-\imath \operatorname{Im} \Pi_{\rho}}\right], \\
& 4\left(\left(\vec{p}_{-} \vec{p}_{+}\right)^{2}-\vec{p}_{-}^{2} \vec{p}_{+}^{2}\right)=\left[\left(\sqrt{s}-E_{+}-E_{-}\right)^{2}+m_{\pi}^{2}-E_{-}^{2}-E_{+}^{2}\right]^{2} \\
& \quad-4\left(E_{+}^{2}-m_{\pi}^{2}\right)\left(E_{-}^{2}-m_{\pi}^{2}\right),
\end{aligned}
$$

where $\theta(x)=1$ for $x>0$ and $\theta(x)=0$ otherwise, $E_{ \pm}=\sqrt{\vec{p}_{ \pm}^{2}+m_{\pi}^{2}}$, and region of integration is restricted by

$$
\left\{\begin{array}{c}
m_{\pi} \leq E_{ \pm} \leq \sqrt{s}-2 m_{\pi} \\
E_{+}+E_{-} \leq \sqrt{s}-m_{\pi} \\
\vec{p}_{+}{ }^{2} \vec{p}_{-}^{2}-\left(\vec{p}_{+} \vec{p}_{-}\right)^{2}>0
\end{array}\right.
$$

Choosing $p_{\rho}^{2}=p_{\rho^{0}}^{2}, p_{\rho^{+}}^{2}, p_{\rho^{-}}^{2}$ allows one to include all three possible charge states of the most complicated diagram for $\omega$ meson in Fig. 1:

$$
\begin{aligned}
& p_{\rho^{0}}^{2}=-s+m_{\pi}^{2}+2 \sqrt{s}\left(E_{+}+E_{-}\right), \\
& p_{\rho^{ \pm}}^{2}=s+m_{\pi}^{2}-2 \sqrt{s} E_{\mp} .
\end{aligned}
$$

In order to restrict the fast growth of the partial widths with $s$ we introduce cut-off FF's for the vertices describing vector-meson decay into two pseudoscalars and pseudoscalar-vector. For the particular form of these FF we choose [16]

$$
\begin{aligned}
C_{V V P}\left(s, r_{0}\right) & =\frac{1+\left(r_{0} m_{V}\right)^{2}}{1+\left(r_{0} \sqrt{s}\right)^{2}}, \\
C_{V P P}\left(s, r_{0}\right) & =\sqrt{\frac{1+\left(r_{0} q_{P}\left(m_{V}^{2}\right)\right)^{2}}{1+\left(r_{0} q_{P}(s)\right)^{2}}} \\
q_{P}(s) & \equiv \sqrt{s-4 m_{P}^{2}}, \quad r_{0}=(2.5 \pm 1.1 \pm 0.5) \mathrm{GeV}^{-1} .
\end{aligned}
$$


Here $r_{0}$ stands for vector-meson effective radius which is taken the same for all decays. With this $\mathrm{FF} \Gamma(s) \rightarrow$ const as $s \rightarrow \infty$. All the above expressions for the self-energy operators are multiplied by the corresponding $\mathrm{FF}$ squared, i.e. $\operatorname{Im} \Pi \ldots(s) \Rightarrow \operatorname{Im} \Pi \ldots(s) C_{\ldots}^{2}\left(s, r_{0}\right)$.

\section{B. Electromagnetic vertex modification}

In this subsection we focus on the photon vector-meson vertices, which follow from (2), (8), (13) and (15). As mentioned in subsection $\amalg A$ the EM couplings $f_{V}(s)$ acquire energy dependence due to loop corrections in the present model.

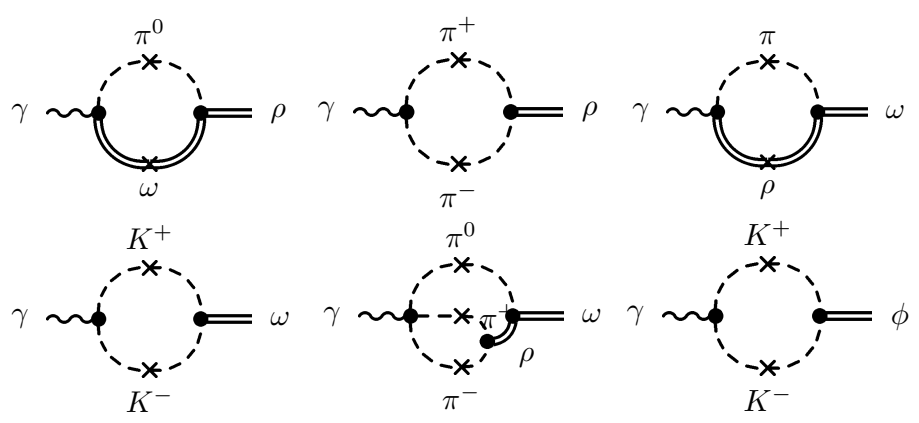

Figure 2: Loops for EM vertex modification.

To be consistent with the approximation for the self-energy we include only the imaginary part of the loop contributions to the EM vertex functions which are shown in Fig. 2 .

In numerical calculation the following formulae are used:

$$
\begin{aligned}
& \operatorname{Im} \Pi_{\gamma\left(\pi^{0} \omega\right) \rho}(s)=\frac{\sqrt{2} e h_{V}}{\sigma_{V}} \operatorname{Im} \Pi_{\rho\left(\pi^{0} \omega\right) \rho}(s), \\
& \operatorname{Im} \Pi_{\gamma(\pi \pi) \rho}(s)=\quad \frac{e}{g} \operatorname{Im} \Pi_{\rho(\pi \pi) \rho}(s), \\
& \operatorname{Im} \Pi_{\gamma\left(\pi^{0} \rho\right) \omega}(s)=\frac{\sqrt{2} e h_{V}}{3 \sigma_{V}} \operatorname{Im} \Pi_{\omega\left(\pi^{0} \rho\right) \omega}(s), \\
& \operatorname{Im} \Pi_{\gamma(K K) \omega}(s)=\quad \frac{e}{g} \operatorname{Im} \Pi_{\omega(K K) \omega}(s), \\
& \operatorname{Im} \Pi_{\gamma(K K) \phi}(s)=-\frac{e}{\sqrt{2} g} \operatorname{Im} \Pi_{\phi(K K) \phi}(s), \\
& \operatorname{Im} \Pi_{\gamma(3 \pi, \pi \rho) \omega}(s)=\frac{e}{24 \sqrt{2} \pi^{2} \theta_{V}} \operatorname{Im} \Pi_{\omega(3 \pi, \pi \rho) \omega}(s) .
\end{aligned}
$$

These expressions should also be multiplied by cut-off $\mathrm{FF} C_{V \gamma P}\left(s, r_{0}\right)$, which is chosen equal to $C_{V V P}\left(s, r_{0}\right)$ in (33).

Fig. 3 illustrates the model for the FF's including self-energy and EM vertex loop corrections. The diagrams in Fig. 3 can also be re-arranged in a different way. As an example, selecting in Fig. 3 contribution from $\rho \pi \pi$ vertex by taking $V=\rho$ and $P=\pi$ one obtains Fig. 4. This figure shows 


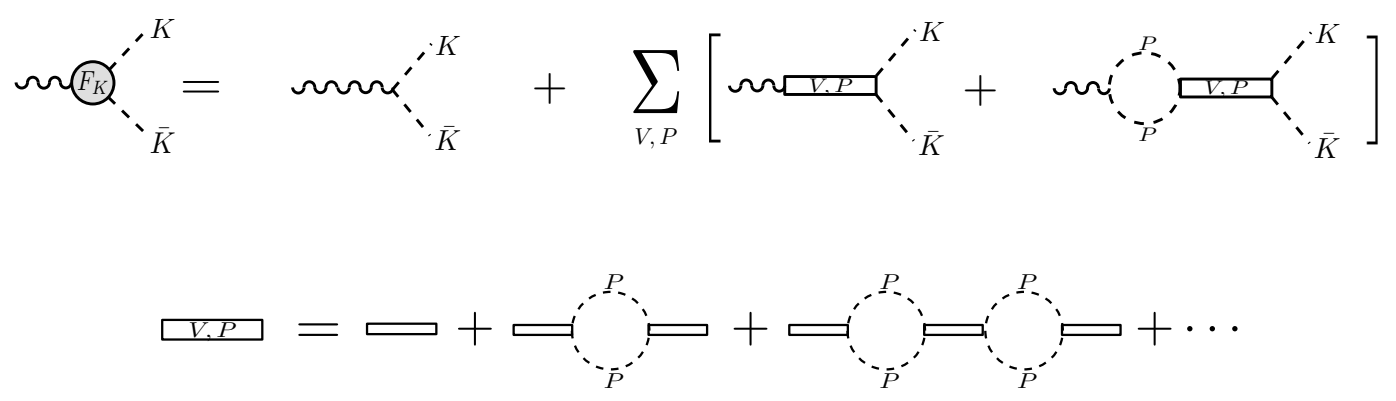

Figure 3: Electromagnetic form factor of $K^{+}\left(K^{-}\right)$.

that the kaon FF contains the pion EM vertex $F^{(\pi)}$ as a building block. For the on-mass-shell pions $F^{(\pi)}$ would reproduce the pion $\mathrm{FF}$.

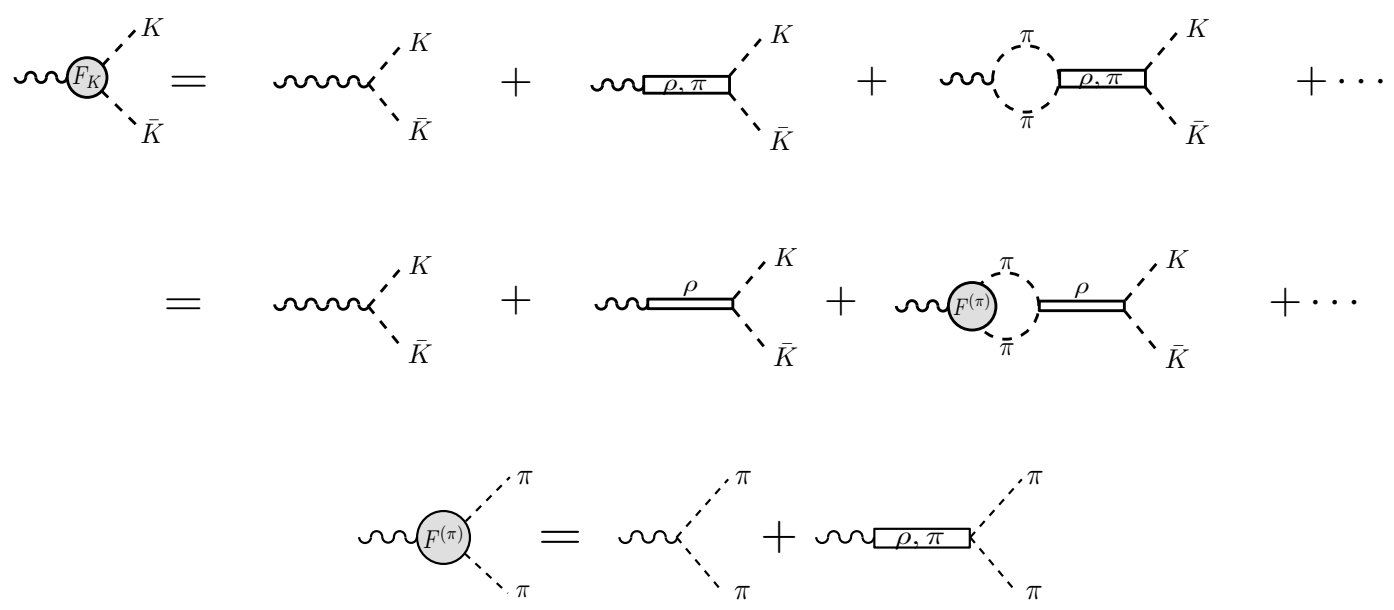

Figure 4: Representation of kaon form factor in terms of pion electromagnetic vertex.

The equations for the modified EM couplings read in terms of the loop corrections

$$
\frac{1}{f_{V}(s)}=\frac{1}{f_{V}^{(0)}}-\frac{\imath}{e s} \sum_{c} \operatorname{Im}_{\gamma(c) V}(s),
$$

for $V=\rho^{0}, \omega, \phi$, and where index $c=\left(\pi^{0} \omega, \pi \pi, \pi^{0} \rho, K K, 3 \pi, 3 \pi-\rho \pi\right)$ stands for the diagrams shown in Fig. 2. The "bare" constants $f_{V}^{(0)}$ are real-valued, i.e. $\operatorname{Im} f_{V}^{(0)}=0$. The modified couplings $f_{V}(s)$ at $s=m_{V}^{2}$ have to describe the leptonic decay widths of the vector mesons

$$
\left|f_{V}\left(s=m_{V}^{2}\right)\right|^{2}=\frac{4}{3} \pi \alpha^{2} \frac{m_{V}}{\Gamma\left(V \rightarrow e^{+} e^{-}\right)}
$$

This allows us to find the bare couplings

$$
\frac{1}{\left(f_{V}^{(0)}\right)^{2}}=\frac{1}{\left|f_{V}\left(s=m_{V}^{2}\right)\right|^{2}}-\frac{1}{e^{2} m_{V}^{4}}\left(\sum_{c} \operatorname{Im}_{\gamma(c) V}\left(s=m_{V}^{2}\right)\right)^{2} .
$$


Using the particle properties [14] (see also Table I) we obtain

$$
f_{\rho}^{(0)}=5.0261, \quad f_{\omega}^{(0)}=17.0601, \quad f_{\phi}^{(0)}=-13.3824
$$

and for arbitrary $s$ the real and imaginary parts of the momentum-dependent couplings $f_{V}(s)$ are calculated from (36).
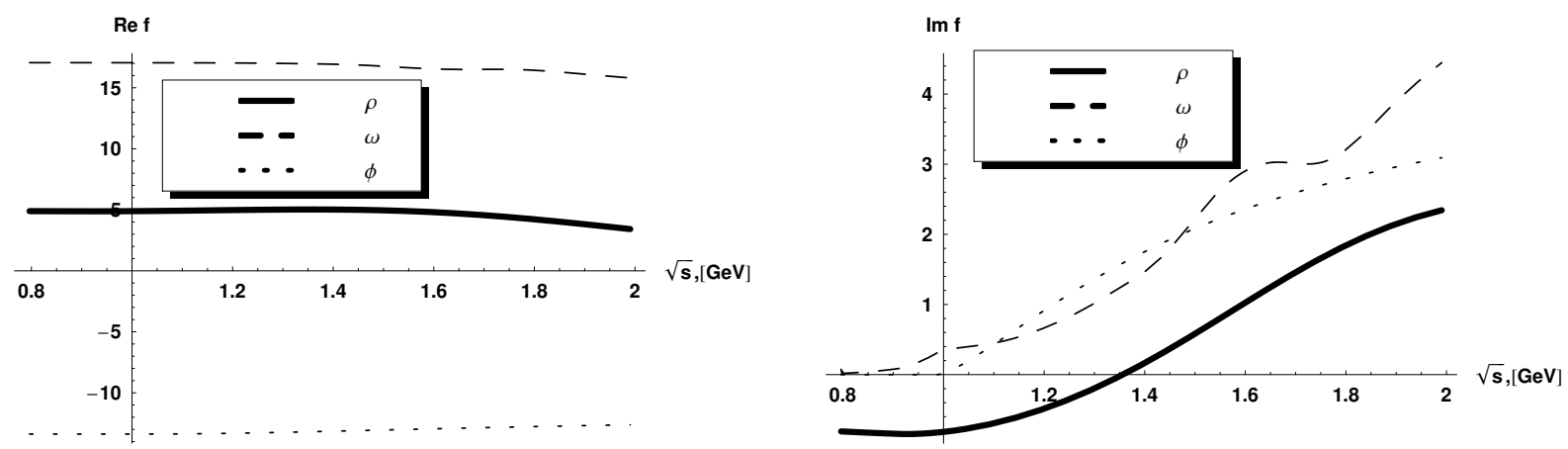

Figure 5: Real (left) and imaginary (right) parts of the coupling constants $f_{V}(s)$ for $\rho, \omega$ and $\phi$ mesons.

On the mass shells the imaginary parts $\operatorname{Im}_{\gamma(c) \omega}\left(s=m_{\omega}^{2}\right)$ and $\operatorname{Im}_{\gamma(c) \phi}\left(s=m_{\phi}^{2}\right)$ turn out to be small and the corresponding bare couplings $f_{\omega}^{(0)}$ and $f_{\phi}^{(0)}$ are very close to the values $\operatorname{Re} f_{\omega}\left(s=m_{\omega}^{2}\right)$ and $\operatorname{Re} f_{\phi}\left(s=m_{\phi}^{2}\right)$. Nevertheless, off the mass shells there remains certain $s$-dependence (see Fig. (5).

\section{Contribution from higher resonances}

The contribution from the higher resonances $\rho^{\prime}, \omega^{\prime}, \phi^{\prime}$ is included by adding

$$
\Delta F_{K^{+}}(s)=-\sum_{V^{\prime}=\rho^{\prime}, \omega^{\prime}, \phi^{\prime}} \frac{g_{V^{\prime} K^{+} K^{-}}}{f_{V^{\prime}}(s)} A_{V^{\prime}}(s), \quad \Delta F_{K^{0}}(s)=-\sum_{V^{\prime}=\rho^{\prime}, \omega^{\prime}, \phi^{\prime}} \frac{g_{V^{\prime} K^{0} \bar{K}^{0}}}{f_{V^{\prime}}(s)} A_{V^{\prime}}(s),
$$

to FF's in (23).

Though the current experimental data are not sufficient to unambiguously find the couplings $f_{V^{\prime}}, g_{V^{\prime} K^{+} K^{-}}$and $g_{V^{\prime} K^{0} \bar{K}^{0}}$, there is a way to constrain them. One can use the branching ratios [14]

$$
\begin{aligned}
\frac{\Gamma\left(\rho(1450) \rightarrow \pi^{+} \pi^{-}\right) \times \Gamma\left(\rho(1450) \rightarrow e^{+} e^{-}\right)}{\Gamma_{\text {tot }}(\rho(1450))} & =\frac{1}{\Gamma_{t o t}\left(\rho^{\prime}\right)} \frac{g_{\rho^{\prime} \pi \pi^{\prime}}^{2} m_{\rho^{\prime}}}{3 \cdot 16 \pi}\left(1-4 \frac{m_{\pi}^{2}}{m_{\rho^{\prime}}^{2}}\right)^{3 / 2} \frac{e^{4} m_{\rho^{\prime}}}{12 \pi f_{\rho^{\prime}}^{2}} \\
& =0.12 \mathrm{keV}, \\
& =\left(\begin{array}{c}
0.027 \\
+0.015 \\
-0.010
\end{array}\right) \mathrm{keV}
\end{aligned}
$$




\begin{tabular}{|c|c|c|c|c|}
\hline Ratios & eqs.(40a), (41) & eqs.(40b), (41) & constrained fit [6] & unconstrained fit [6] \\
\hline$\frac{g_{\rho^{\prime} K^{+} K^{-}}}{f_{\rho^{\prime}}}$ & $-0.063 \pm 0.005$ & $-0.03 \pm 0.0024 \pm 0.007$ & $-\frac{0.112}{1.195} \frac{g_{\rho K^{+} K^{-}}}{f_{\rho}}=-0.056$ & $-\frac{0.124}{1.139} \frac{g_{\rho K^{+} K^{-}}}{f_{\rho}}=-0.065$ \\
\hline$\frac{g_{\omega^{\prime} K^{+} K^{-}}}{f_{\omega^{\prime}}}$ & $\frac{g_{\rho^{\prime} K^{+} K^{-}}}{3 f_{\rho^{\prime}}}$ & $\frac{g_{\rho^{\prime} K^{+} K^{-}}}{3 f_{\rho^{\prime}}}$ & $-\frac{0.112}{1.195} \frac{g_{\omega K^{+} K^{-}}}{f_{\omega}}=-0.016$ & $-\frac{0.018}{1.467} \frac{g_{\omega K^{+} K^{-}}}{f_{\omega}}=-0.002$ \\
\hline$\frac{g_{\phi^{\prime} K^{+} K^{-}}}{f_{\phi^{\prime}}}$ & $-0.036 \pm 0.012 \pm 0.008$ & $-0.036 \pm 0.012 \pm 0.008$ & $-\frac{0.018}{1.018} \frac{g_{\phi K^{+} K^{-}}}{f_{\phi}}=-0.005$ & $-\frac{0.001}{0.999} \frac{g_{\phi K^{+} K^{-}}}{f_{\phi}} \approx 0.0$ \\
\hline
\end{tabular}

Table IV: Ratios of coupling constants for resonances $\rho^{\prime}, \omega^{\prime}, \phi^{\prime}$ (only couplings to $K^{+} K^{-}$are shown for brevity). Errors are due to uncertainty in decay widths (first) and branching ratios (second). The signs of couplings are chosen opposite to those for $\rho, \omega, \phi$.

$$
\begin{aligned}
\frac{\Gamma\left(\phi(1680) \rightarrow K_{L}^{0} K_{S}^{0}\right) \times \Gamma\left(\phi(1680) \rightarrow e^{+} e^{-}\right)}{\Gamma_{t o t}(\phi(1680))^{2}} & =\frac{1}{\Gamma_{t o t}\left(\phi^{\prime}\right)^{2}} \frac{g_{\phi^{\prime} K^{0} \bar{K}^{0}}^{2} m_{\phi^{\prime}}}{3 \cdot 16 \pi}\left(1-4 \frac{m_{K}^{2}}{m_{\phi^{\prime}}^{2}}\right)^{3 / 2} \frac{e^{4} m_{\phi^{\prime}}}{12 \pi f_{\phi^{\prime}}^{2}} \\
& =(0.131 \pm 0.059) \times 10^{-6}
\end{aligned}
$$

and total widths $\Gamma_{t o t}\left(\rho^{\prime}\right)=400 \pm 60 \mathrm{MeV}, \Gamma_{\text {tot }}\left(\omega^{\prime}\right)=215 \pm 35 \mathrm{MeV}$ and $\Gamma_{\text {tot }}\left(\phi^{\prime}\right)=150 \pm 50 \mathrm{MeV}$.

From these relations one finds ratios of the strong and EM couplings, $g_{\rho \prime K^{+} K^{-}} / f_{\rho \prime}$ and $g_{\phi \prime K^{0} \bar{K}^{0}} / f_{\phi \prime}$. We can also use the $S U(3)$ relations for the ratios (see Tables 【 and II):

$$
\begin{aligned}
\frac{g_{\rho \prime K^{+} K^{-}}}{f_{\rho \prime}}: \frac{g_{\omega \prime K^{+} K^{-}}}{f_{\omega \prime}}: \frac{g_{\phi \prime K^{+} K^{-}}}{f_{\phi \prime}} & =\frac{1}{2}: \frac{1}{6}: \frac{1}{3}, \\
\frac{g_{\rho \prime K^{0} \bar{K}^{0}}}{f_{\rho \prime}}: \frac{g_{\omega \prime K^{0} \bar{K}^{0}}}{f_{\omega \prime}}: \frac{g_{\phi \prime K^{0} \bar{K}^{0}}}{f_{\phi \prime}} & =-\frac{1}{2}: \frac{1}{6}: \frac{1}{3}
\end{aligned}
$$

and $\left|g_{\rho \prime K^{+} K^{-}}\right|=\left|g_{\rho^{\prime} K^{0} \bar{K}^{0}}\right|$.

From (40a) and (41) we get the 2nd column of Table IV. It is seen that $g_{\rho \prime K^{+} K^{-}} / f_{\rho \prime}$ and $g_{\phi \prime K^{+} K^{-}} / f_{\phi \prime}$ approximately follow $S U(3)$ symmetry (42) that allows us to obtain parameters for $\omega^{\prime}$-meson. Note that $S U(3)$ symmetry is not satisfied for parameters calculated from (40b) and (41), which are shown in the 3rd column of Table IV. Above estimates for parameters of the "primed" resonances are used further in calculations. For comparison in the 4th and 5th columns we present the values obtained from the "constrained" and "unconstrained" fits to data in Ref. [6].

There still remains a sign ambiguity in these ratios. One may determine the relative sign of the $\rho^{\prime}, \omega^{\prime}, \phi^{\prime}$ contribution with respect to the $\rho, \omega, \phi$ one using the following reasoning. On the basis of quark counting rule in perturbative QCD [24] the FF behaves like $F_{K^{+}}(s) \rightarrow a / s$ at $s \rightarrow-\infty$, where $a=-16 \pi F_{\pi}^{2} \alpha_{s}(s)$. We obtain in this limit from (23) and (39)

$$
\begin{aligned}
& F_{K^{+}}(s) \underset{s \rightarrow-\infty}{\longrightarrow} b+\frac{a}{s}, \\
& b=1-\sum_{V=\rho, \omega, \phi} \frac{g_{V K^{+} K^{-}}}{f_{V}}-\sum_{V^{\prime}=\rho^{\prime}, \omega^{\prime}, \phi^{\prime}} \frac{g_{V^{\prime} K^{+} K^{-}}}{f_{V^{\prime}}}, \\
& a=-\sum_{V=\rho, \omega, \phi} \frac{g_{V K^{+} K^{-} m_{V}^{2}}}{f_{V}}-\sum_{V^{\prime}=\rho^{\prime}, \omega^{\prime}, \phi^{\prime}} \frac{g_{V^{\prime} K^{+} K^{-} m_{V^{\prime}}^{2}}}{f_{V^{\prime}}} .
\end{aligned}
$$


For the correct asymptotic behavior the constant $b$ in (44) should be equal to zero. One can check however that the contribution from the lowest resonances $\rho, \omega, \phi$ with the couplings $g_{V K^{+} K^{-}} / f_{V}$ taken from experiment does not lead to vanishing of $b$. If we add the contribution from the higher resonances $\rho^{\prime}, \omega^{\prime}, \phi^{\prime}$ choosing the negative relative sign of couplings $g_{V^{\prime} K^{+} K^{-}} / f_{V^{\prime}}$ with respect to $g_{V K^{+} K^{-}} / f_{V}$, then the constant $b$ becomes closer to zero. In this way the asymptotic behavior of the form factors is partially improved. The sign "minus" for the higher resonances also improves significantly description of the experimental form factors which are calculated in sect. IV] Note also that the constant $a$ in (45) takes negative values with the parameters in Table IV.

There is also experimental information on the relative phases of the higher resonance contribution in a different reaction $e^{+} e^{-} \rightarrow \pi^{+} \pi^{-} \pi^{0}[16,25]$. Our choice of the relative sign is in line with the findings of these papers.

\section{RESULTS OF CALCULATION}

\section{A. Kaon form factors and cross section of $e^{+} e^{-} \rightarrow K \bar{K}$ reaction}

In this section we present results for the charged and neutral kaons. The FF's are calculated from (26), (27), (39), and the $e^{+} e^{-}$annihilation cross section from (11). Figs. 6 and 7 show FF in the time-like region, while Fig. 11] shows FF in the space-like region.

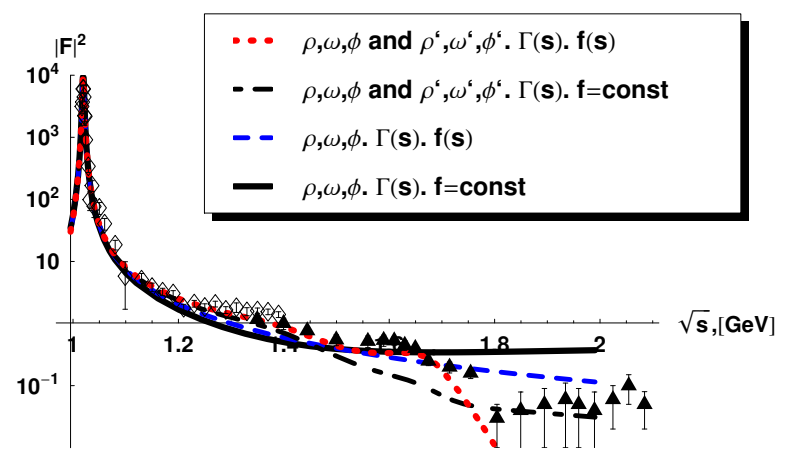

Figure 6: Charged kaon EM form factor in the time-like region. Data: diamonds (open) are from [26], triangles - from [27].

The solid curves (see legends in the plots) represent a simple VMD-like model in which only $\rho$, $\omega$ and $\phi$ resonances are included. The meson widths are taken $s$-dependent (see sect. IIIA) while the couplings of vector mesons to photon $f_{V}$ are independent of momentum. As known, such a model can describe experiment only in vicinity of the $\phi(1020)$ resonance. 


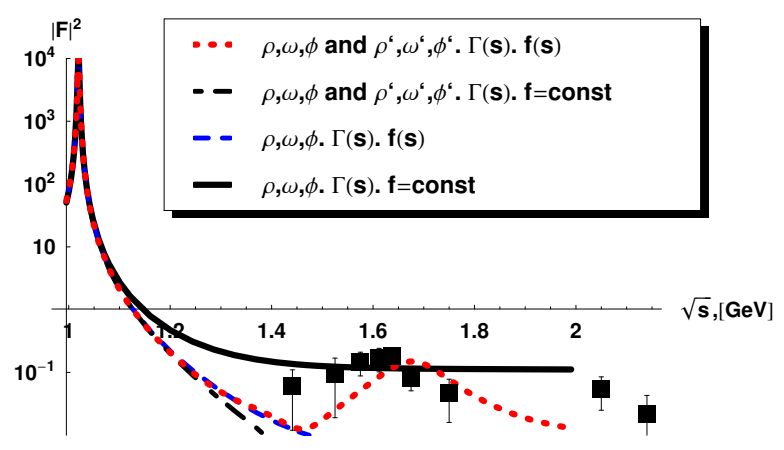

Figure 7: Neutral kaon EM form factor in the time-like region. Data (boxes) are from [28].
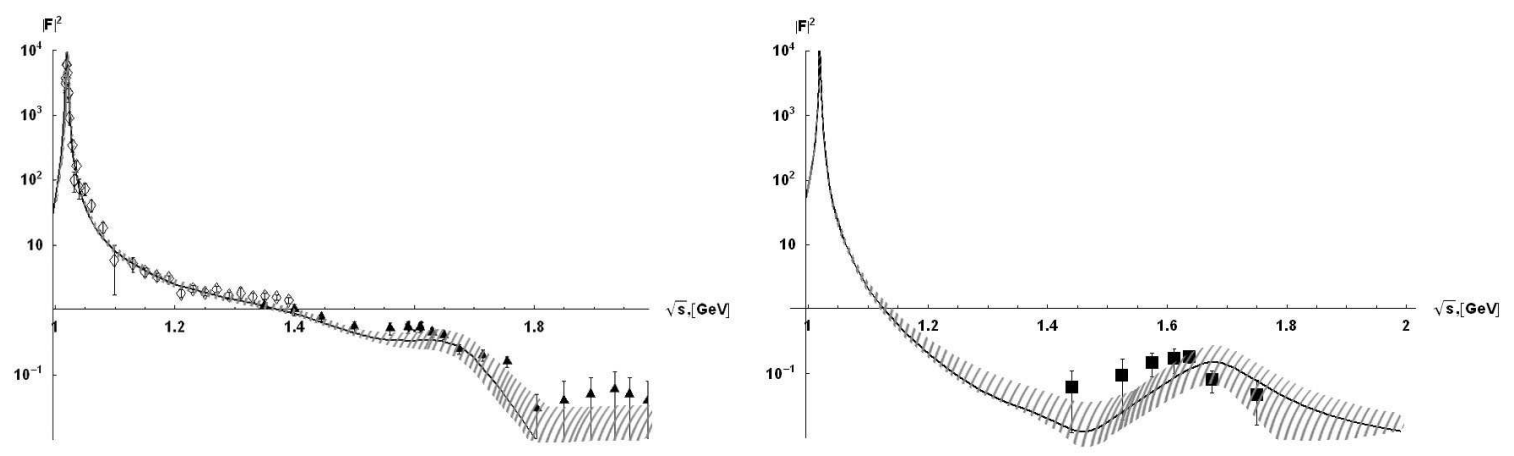

Figure 8: Form factors of charged kaon (left) and neutral kaon (right). Hatched area shows error corridor caused by uncertainties in parameters of vector mesons. Data are the same as in Figs. 6 and 7 .

The long-dashed curves include in addition the momentum-dependent EM couplings. It is seen that this inclusion does not improve considerably the description. These results suggest the need for further complication of the model.

Taking into consideration the excited resonances $\rho^{\prime}, \omega^{\prime}$ and $\phi^{\prime}$ with momentum-dependent widths and constant couplings $f_{V}$ we obtain the dot-dashed curves. The corresponding calculation improves the agreement with available data in comparison with the simple VMD model. The contribution from $\rho(1450), \omega(1420)$ and $\phi(1680)$ resonances is noticeable.

The short-dashed curves represent the most advanced calculation. These curves include the momentum-dependent widths for all intermediate states, "dressed" EM vertices (for $\rho, \omega$ and $\phi$ resonances) and cut-off FF's in the self-energies and EM vertices (see (33) and (34)). We have not attempted to develop vertex "dressing" for the higher resonances due to the present experimental uncertainties in their decay rates. We should note that the authors of Ref. [6] also obtained a good description of the data by fixing the values of the parameters $f_{V}$ from the fit. In our procedure of "dressing" the couplings a reasonable agreement is achieved without fitting the parameters. 


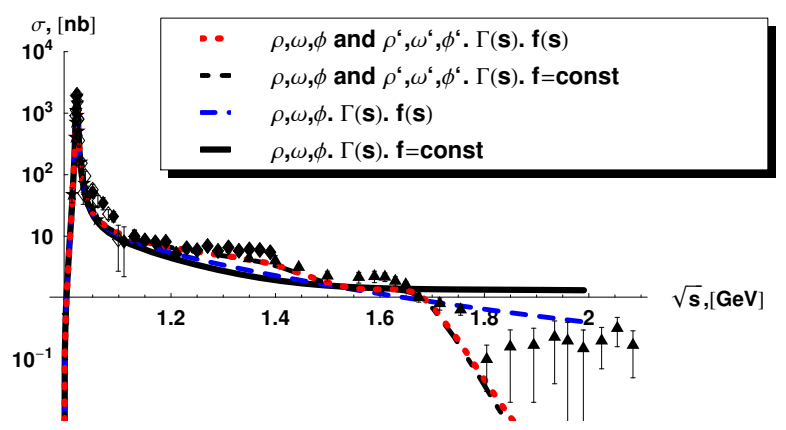

Figure 9: Total cross section of $e^{+} e^{-}$annihilation into charged kaons. Data: stars are from [29], diamonds (filled) - from [30], triangles - from [27], diamonds (open) - from [26].

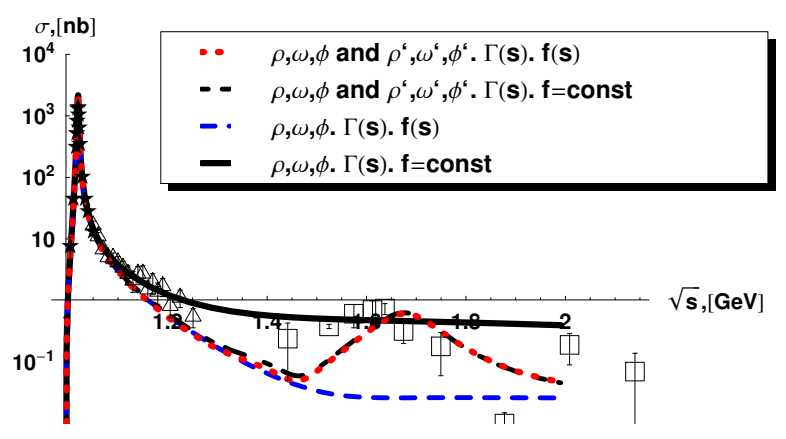

Figure 10: Total cross section of $e^{+} e^{-}$annihilation into neutral kaons. Data: stars are from [29], triangles (open) - from [4], boxes (open) - from [28].

It is of interest to study sensitivity of calculated FF's to uncertainties in the model parameters. The uncertainties in parameters $f_{V}$ and $g_{V^{\prime} K K} / f_{V^{\prime}}$ for vector mesons (see Tables $\Pi$ and IV) arises due to a limited experimental accuracy in decay widths and branching ratios, especially for the primed resonances. Fig. 8 demonstrates how these model uncertainties influence FF's.

Figs. 9 and 10 represent the $e^{+} e^{-} \rightarrow K \bar{K}$ cross section. They are compared with all available data. Finally the plot in Fig. 11 shows the charged kaon FF in the space-like region of photon momentum. This figure demonstrates good agreement with data [31]. At the same time, the FF in the region of $-q^{2}<0.16 \mathrm{GeV}^{2}$ is not sensitive to ingredients of the model.

Note that kaon FF's in the space-like region have been studied earlier in various approaches: quark Dyson-Schwinger equation [32], ChPT in order $\mathcal{O}\left(p^{6}\right)$ [33], quark linear $\sigma$-model [34], model based on pion FF and data on $K^{+} \rightarrow \pi^{+} e^{+} e^{-}$[35], relativistic constituent quark model [36].

From Figs. 6]-10 one can conclude that the developed model is consistent with the experimental 


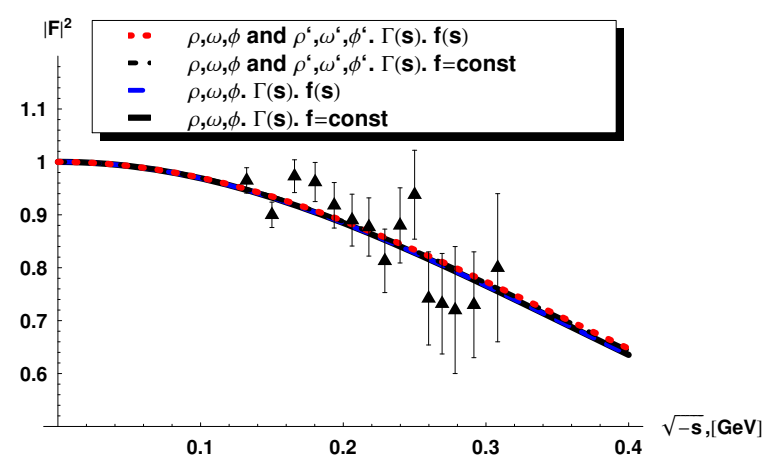

Figure 11: Charged kaon EM form factor in the space-like region. Data are from [31].

information [4], 26, 27, 28, 29, 30] in the 1-1.75 GeV energy region. Although the most important contributions are included in the model, some deviations show up in the higher-energy region. They may be attributed to other intermediate states or experimental uncertainty in the parameters of $\rho^{\prime}, \omega^{\prime}$ and $\phi^{\prime}$ resonances.

\section{B. Contribution of $K^{+} K^{-}$and $K^{0} \bar{K}^{0}$ channels to anomalous magnetic moment of muon}

The contribution of the $K \bar{K}$-channel to $a_{\mu}$ is directly related to $F_{K^{+}}(s)$ and $F_{K^{0}}(s)$ via the dispersion integral [37] (see also [38]):

$$
\begin{aligned}
a_{\mu}^{h a d, K \bar{K}} & =\frac{\alpha^{2}}{3 \pi^{2}} \int_{4 m_{K}^{2}}^{\infty} W(s) R(s) \frac{\mathrm{d} s}{s}, \\
W(s) & =\int_{0}^{1} \frac{x^{2}(1-x)}{x^{2}+(1-x) s / m_{\mu}^{2}} \mathrm{~d} x \\
& =y^{2}\left(1-\frac{y^{2}}{2}\right)+(1+y)^{2}\left(1+\frac{1}{y^{2}}\right)\left[\ln (1+y)-y+\frac{y^{2}}{2}\right]+\frac{1+y}{1-y} y^{2} \ln y,
\end{aligned}
$$

where $y=(1-\beta) /(1+\beta), \beta=\left(1-4 m_{\mu}^{2} / s\right)^{1 / 2}, m_{\mu}$ is the muon mass, and $R(s)$ is the ratio of the cross sections

$$
R(s)=\frac{\sigma\left(e^{+} e^{-} \rightarrow K \bar{K}\right)}{\sigma\left(e^{+} e^{-} \rightarrow \mu^{+} \mu^{-}\right)}=\frac{\left(1-\frac{4 m_{K}^{2}}{s}\right)^{3 / 2}}{4\left(1+2 \frac{m_{\mu}^{2}}{s}\right)\left(1-\frac{4 m_{\mu}^{2}}{s}\right)^{1 / 2}}\left|F_{K}(s)\right|^{2} .
$$

Here $\sigma\left(e^{+} e^{-} \rightarrow K \bar{K}\right)$ is the 'bare' (lowest order) cross section, not including initial-state radiation, $\gamma e^{+} e^{-}$vertex corrections and vacuum polarization effects.

The calculated values are presented in Table $\nabla$ together with the inaccuracy caused by uncertainty in the parameters of the model. The $K \bar{K}$-channels contribute $(34.70 \pm 1.01) \times 10^{-10}$ to the hadronic contribution $a_{\mu}^{\text {had }}$. The latter, according to Ref. [38], is $a_{\mu}^{\text {had,LO }}=\left(696.3 \pm 6.2_{\exp } \pm\right.$ 


\begin{tabular}{|c|c|c|c|}
\hline & $K^{+} K^{-}$ & $K^{0} \bar{K}^{0}$ & total $K \bar{K}$ \\
\hline calculation based on (40a), (41) & $19.18 \pm 0.45$ & $15.60 \pm 0.40$ & $34.78 \pm 0.85$ \\
\hline calculation based on (40b), (41) & $19.00 \pm 0.51$ & $15.70 \pm 0.39$ & $34.70 \pm 0.90$ \\
\hline average & $19.06 \pm 0.57$ & $15.64 \pm 0.44$ & $34.70 \pm 1.01$ \\
\hline
\end{tabular}

Table V: Contribution of $K \bar{K}$-channels to anomalous magnetic moment of the muon $a_{\mu}^{\text {had, } K \bar{K}}$ in units $10^{-10}$.

$\left.3.6_{\text {rad }}\right) \times 10^{-10}$ (in LO). We can compare our result with that of [38] for the $K \bar{K}$-channels (see Table 1 in [38], entries for $\phi$ and $K \bar{K}$ contributions in $e^{+} e^{-}$annihilation):

$$
\begin{aligned}
& \left(35.71 \pm 0.84_{\text {exp }} \pm 0.20_{\text {rad }}\right)_{\phi} \times 83.1 \%+\left(4.63 \pm 0.40_{\text {exp }} \pm 0.06_{\text {rad }}\right)_{K^{+} K^{-}} \\
& +\left(0.94 \pm 0.10_{\text {exp }} \pm 0.01_{\text {rad }}\right)_{K_{S} K_{L}}=35.24 \pm 1.20_{\text {exp }} \pm 0.24_{\text {rad }}
\end{aligned}
$$

in units $10^{-10}$. It is seen that our model gives $a_{\mu}^{h a d, K \bar{K}}$ very close to the value obtained from $e^{+} e^{-}$ annihilation cross sections.

\section{CONCLUSIONS}

We developed a model for electromagnetic FF's of the charged and neutral kaon in the time-like $(\sqrt{s}=1-2 \mathrm{GeV})$ and space-like $(s \leq 0)$ regions of the photon momentum. The model is based on the chiral perturbation theory (ChPT) with vector mesons [7]. The photon vector-meson vertices by construction depend on the photon momentum $q$ and are explicitly gauge invariant. This ensures the correct normalization of FF's at the real-photon point $F_{K^{+}}\left(q^{2}=0\right)=1$ and $F_{K^{0}}\left(q^{2}=0\right)=0$ without need for parameters adjustment.

Beyond the tree level the model includes certain loop corrections, such as self-energy polarization operators in vector-meson propagators, and "dressed" photon-meson vertices. For construction of the vertices we apply chiral Lagrangians [7, 12, 13] and WZW Lagrangians [9, 10]. The coupling constants are fixed from experimental decay widths of resonances. The parameters in the oddintrinsic-parity sector are also compared with those obtained from a generalization of the WZW approach for vector and axial-vector mesons [13] and extended Nambu-Jona-Lasinio model [12].

Comparison of the calculations with available data for the $\mathrm{FF}$ 's and $e^{+} e^{-}$annihilation cross sections indicates that the model is consistent with experimental information in the $\sqrt{s}=1-1.75$ $\mathrm{GeV}$ energy region. Using the dressed photon-meson vertices and adding the resonances $\rho^{\prime}=$ $\rho(1450), \omega^{\prime}=\omega(1420)$ and $\phi^{\prime}=\phi(1680)$ considerably improve description of the data. A reasonable 
agreement is achieved without fitting the parameters of the model.

Although the most important contributions are included, deviations from the data appear at high energies, $\sqrt{s} \sim 2 \mathrm{GeV}$. Those may be attributed to missing contribution from the higher ("double-primed") resonances $\rho(1700)$ and $\omega(1650)$ [14], or possibly experimental uncertainty in parameters of the $\rho^{\prime}, \omega^{\prime}$ and $\phi^{\prime}$.

The charged kaon FF extended to the space-like region agrees with the data [31] at relatively small values of momentum transfer, $-q^{2}<0.16 \mathrm{GeV}^{2}$, though all variants of the model lead to the close results. Current measurements at JLab [39] of the longitudinal cross section in the reactions $e p \rightarrow e \Lambda K^{+}$and $e p \rightarrow e \Sigma^{0} K^{+}$will provide information on kaon FF at momentum transfer up to $-q^{2} \sim 3 \mathrm{GeV}^{2}$. These experiments can help to discriminate between variants of the present model, as well as between various theoretical approaches [32, 33, $\underline{34}, \underline{35}, \underline{36}]$.

The calculated FF's allowed us also to evaluate leading-order contribution of the $K \bar{K}$-channel to the muon anomalous magnetic moment $a_{\mu}$. The calculated value $a_{\mu}^{\text {had, } K^{+} K^{-}+K^{0} \bar{K}^{0}}=(34.70 \pm$ 1.01) $\times 10^{-10}$ is in agreement with result [38] obtained from the experimental $e^{+} e^{-}$annihilation cross sections.

\section{Acknowledgement}

We would like to thank S. Eidelman and N. Merenkov for useful suggestions and remarks. This work is supported by grant INTAS Ref. Nr 05-1000008-8328.

\section{Appendix A: CHIRAL LAGRANGIAN FOR PSEUDOSCALAR, VECTOR MESONS AND PHOTON}

In the even intrinsic-parity sector we choose $\mathcal{O}\left(p^{2}\right)$ chiral Lagrangian derived by Ecker et al. [7], which includes interaction of pseudoscalar, polar-vector, axial-vector mesons and photon. We omit in this Lagrangian contribution from axial-vector mesons which is not relevant here. The explicit form is then

$$
\begin{aligned}
L= & \frac{F_{\pi}^{2}}{4} \operatorname{Tr}\left(D_{\mu} U D^{\mu} U^{\dagger}+\chi U^{\dagger}+\chi^{\dagger} U\right)-\frac{1}{4} F_{\mu \nu} F^{\mu \nu} \\
& -\frac{1}{2} \operatorname{Tr}\left(\nabla^{\lambda} V_{\lambda \mu} \nabla_{\nu} V^{\nu \mu}-\frac{1}{2} M_{V}^{2} V_{\mu \nu} V^{\mu \nu}\right) \\
& +\frac{F_{V}}{2 \sqrt{2}} \operatorname{Tr}\left(V_{\mu \nu} f_{+}^{\mu \nu}\right)+\frac{i G_{V}}{\sqrt{2}} \operatorname{Tr}\left(V_{\mu \nu} u^{\mu} u^{\nu}\right),
\end{aligned}
$$


where $U=\exp \left(i \sqrt{2} \Phi / F_{\pi}\right)$, $\Phi$ describes the pseudoscalar mesons, $V_{\mu \nu}$ is antisymmetric field describing the vector mesons, and $F^{\mu \nu}=\partial^{\mu} B^{\nu}-\partial^{\nu} B^{\mu}$ is the EM tensor for the gauge photon field $B^{\mu}$. The pion weak-decay constant $F_{\pi}$ and coupling constants $F_{V}, G_{V}$ are specified in sect. III.

The covariant derivative $D_{\mu}$ is defined as

$$
D_{\mu} U=\partial_{\mu} U+\imath e B_{\mu}[U, Q]
$$

with quark charge matrix $Q=\operatorname{diag}\left(\frac{2}{3},-\frac{1}{3},-\frac{1}{3}\right)=\frac{1}{2} \lambda_{3}+\frac{1}{2 \sqrt{3}} \lambda_{8}, \quad \lambda_{a}(a=1, \ldots, 8)$ are the GellMann matrices and $\lambda_{0}=\sqrt{\frac{2}{3}}$. Also in (A.1) $\chi$ is proportional to quark mass matrix $\mathcal{M}=$ $\operatorname{diag}\left(m_{u}, m_{d}, m_{s}\right)$ and

$$
\begin{aligned}
& U=u^{2}, \quad u^{\mu}=\imath u^{+}\left(D^{\mu} U\right) u^{+}, \\
& f_{ \pm}^{\mu \nu}=e F^{\mu \nu}\left(u Q u^{+} \pm u^{+} Q u\right) .
\end{aligned}
$$

For other definitions see [7].

In the odd intrinsic-parity sector, Lagrangian [11, 12] in the vector formulation for vector mesons is applied. The pseudoscalar-vector interaction of chiral order $\mathcal{O}\left(p^{3}\right)$ and $\mathcal{O}\left(p^{2}\right)$ is

$$
L_{i n t}=i \theta_{V} \epsilon_{\mu \nu \alpha \beta} \operatorname{Tr}\left(V^{\mu} u^{\nu} u^{\alpha} u^{\beta}\right)+h_{V} \epsilon_{\mu \nu \alpha \beta} \operatorname{Tr}\left(V^{\mu}\left\{u^{\nu}, f_{+}^{\alpha \beta}\right\}\right)+\sigma_{V} \epsilon_{\mu \nu \alpha \beta} \operatorname{Tr}\left(V^{\mu}\left\{u^{\nu}, \partial^{\alpha} V^{\beta}\right\}\right)
$$

where $\{a, b\} \equiv a b+b a$ and we omitted piece with axial-vector fields, higher-order terms in vector fields $V^{\mu}$, and some other terms which give no contribution to form factors in the present model. The coupling parameters $\theta_{V}, h_{V}$ and $\sigma_{V}$ are discussed in sect. II.

The $S U(3)$ flavor symmetry of the meson multiplets is conventionally accounted for by using notation for pseudoscalar-meson $\left(J^{P}=0^{-}\right)$octet, and for the vector-meson $\left(J^{P C}=1^{--}\right)$nonet in vector and tensor formulation, respectively

$$
\begin{aligned}
\Phi & =\frac{1}{\sqrt{2}} \sum_{a=1}^{8} \lambda_{a} \phi^{a}, \quad V_{\mu}=\frac{1}{\sqrt{2}} \sum_{a=1}^{8} \lambda_{a} V_{\mu}^{a}+\frac{1}{\sqrt{2}} \lambda_{0} V_{\mu}^{0}, \\
V_{\mu \nu} & =\frac{1}{\sqrt{2}} \sum_{a=1}^{8} \lambda_{a} V_{\mu \nu}^{a}+\frac{1}{\sqrt{2}} \lambda_{0} V_{\mu \nu}^{0} .
\end{aligned}
$$

In the present paper we do not need $K^{* \pm}, K^{* 0}, \overline{K^{* 0}}$ and $\eta$ mesons and it is sufficient to take

$$
\begin{aligned}
\Phi & =\frac{1}{\sqrt{2}}\left(\pi_{1} \lambda_{1}+\pi_{2} \lambda_{2}+\pi_{3} \lambda_{3}+K_{1} \lambda_{4}+K_{2} \lambda_{5}+K_{3} \lambda_{6}+K_{4} \lambda_{7}\right), \\
V^{\mu} & =\frac{1}{\sqrt{2}}\left(\rho_{1}^{\mu} \lambda_{1}+\rho_{2}^{\mu} \lambda_{2}+\rho_{3}^{\mu} \lambda_{3}+\omega^{\mu}\left(\lambda_{0} \sin \theta+\lambda_{8} \cos \theta\right)+\phi^{\mu}\left(\lambda_{0} \cos \theta-\lambda_{8} \sin \theta\right)\right), \\
V^{\mu \nu} & =\frac{1}{\sqrt{2}}\left(\rho_{1}^{\mu \nu} \lambda_{1}+\rho_{2}^{\mu \nu} \lambda_{2}+\rho_{3}^{\mu \nu} \lambda_{3}+\omega^{\mu \nu}\left(\lambda_{0} \sin \theta+\lambda_{8} \cos \theta\right)+\phi^{\mu \nu}\left(\lambda_{0} \cos \theta-\lambda_{8} \sin \theta\right)\right),
\end{aligned}
$$


where the physical fields are defined as

$$
\begin{aligned}
\pi^{ \pm} & =\frac{1}{\sqrt{2}}\left(\pi_{1} \mp \imath \pi_{2}\right), & K^{ \pm} & =\frac{1}{\sqrt{2}}\left(K_{1} \mp \imath K_{2}\right), \\
K^{0} & =\frac{1}{\sqrt{2}}\left(K_{3}-\imath K_{4}\right), & \bar{K}^{0} & =\frac{1}{\sqrt{2}}\left(K_{3}+\imath K_{4}\right), \\
\rho^{ \pm} & =\frac{1}{\sqrt{2}}\left(\rho_{1} \mp \imath \rho_{2}\right), & \rho^{0} & =\rho_{3}, \quad \pi^{0}=\pi_{3} .
\end{aligned}
$$

Mixing angle $\theta$ can be estimated from experiment. It is convenient to use $\omega-\phi$ mixing parameter [2] $\epsilon_{\omega \phi}=\sin \left(\theta_{0}-\theta\right)=0.058$ which naturally appears for OZI-forbidden processes. The "ideal mixing" (with $\sin \theta_{0}=\sqrt{2 / 3}, \cos \theta_{0}=1 / \sqrt{3}$ ) corresponds to quark content of the vector mesons: $\omega=(\bar{u} u+\bar{d} d) / \sqrt{2}$ and $\phi=\bar{s} s$.

[1] R.R. Akhmetshin et al. [CMD-2 Collaboration], Phys. Lett. B 527, 161 (2002); Phys. Lett. B 578, 285 (2004).

[2] F. Klingl, N. Kaiser, and W. Weise, Z. Phys. A 356, 193 (1996).

[3] S.K. Dubinsky, A.Yu. Korchin, and N.P. Merenkov, Zh. Eksp. Teor. Fiz. 126, 259 (2004) arXiv:hep-ph/0407191; JETP 99, 225 (2004);

S. Dubinsky, A. Korchin, N. Merenkov, G. Pancheri, and O. Shekhovtsova, Eur. Phys. J. C 40, 41 (2005) arXiv:hep-ph/0411113.

[4] R.R. Akhmetshin et al., Phys. Lett. B 551, 27 (2003) arXiv:hep-ex/0211004.

[5] M.N. Achasov et al. [SND Collaboration], Preprint Budker INP-2005-35, Novosibirsk, 2005.

[6] C. Bruch, A. Khodjamirian, and J.H. Kühn, Eur. Phys. J. C 39, 41 (2005).

[7] G. Ecker, J. Gasser, A. Pich, and E. de Rafael, Nucl. Phys. B 321, 311 (1989).

[8] G. Ecker, J. Gasser, H. Leutwyler, and A. Pich, Phys. Lett. B 223, 425 (1989).

[9] J. Wess and B. Zumino, Phys. Lett. B 37, 95 (1971).

[10] E. Witten, Nucl. Phys. B 223, 422 (1983).

[11] G. Ecker, A. Pich, and E. de Rafael, Phys. Lett. B 237, 481 (1990).

[12] J. Prades, Z. Phys. C 63491 (1994); Erratum, Eur. Phys. J. C 11, 571 (1999).

[13] O. Kaymakcalan, S. Rajeev, and J. Schechter, Phys. Rev. D 30, 594 (1984);

P. Ko and S. Rudaz, Phys. Rev. D 50, 6877 (1994).

[14] S. Eidelman et al, Review of Particle Physics (Particle Data Group), Phys. Lett. B 592, 1 (2004) (URL: http://pdg.lbl.gov).

[15] P. Jain, R. Johnson, U.G. Meissner, N.W. Park, and J. Schechter, Phys. Rev. D 373252 (1988).

[16] M.N. Achasov et al., Phys. Rev. D 68052006 (2003) arXiv:hep-ex/0305049].

[17] N.N. Achasov and A.A. Kozhevnikov, Phys. Lett. B 233, 474 (1989).

[18] N.N. Achasov and A.A. Kozhevnikov, Phys. Rev. D 52, 3119 (1995). 
[19] A. Aloisio et al., Phys. Lett. B 561, 55 (2003) arXiv:hep-ex/0303016.

[20] M.N. Achasov et al., Nucl. Phys. B 569, 158 (2000) arXiv:hep-ex/9907026.

[21] M.N. Achasov et al., AIP Conf. Proc. 717 (2004) 60 arXiv:hep-ex/0309055.

[22] C. Itzykson and J.-B. Zuber, Quantum Field Theory, McGraw-Hill Book Company, 1980.

[23] O. Scholten, A.Yu. Korchin, V. Pascalutsa, and D. Van Neck, Phys. Lett. B 384, 13 (1996);

A.Yu. Korchin, O. Scholten, and R.G.E. Timmermans, Phys. Lett. B 438, 1 (1998).

[24] G.R. Farrar and D.R. Jackson, Phys. Rev. Lett. 43, 246 (1979);

G.P. Lepage and S.J. Brodsky, Phys. Rev. D 22, 2157 (1980).

[25] M.N. Achasov et al. Phys. Lett. B 462, 365 (1999); Phys. Rev. D 66, 032001 (2002);

[26] P.M. Ivanov et al., Phys. Lett. B 107, 297 (1981).

[27] D. Bisello et al. [DM2 Collaboration], Z. Phys. C 39, 13 (1988).

[28] F. Mane et al. [DM1 Collaboration], Phys. Lett. B 99, 261 (1980).

[29] M.N. Achasov et al. [SND Collaboration], Phys. Rev. D 63, 072002 (2001) arXiv:hep-ex/0009036].

[30] S.I. Dolinsky et al., Phys. Rep. 202, 99 (1991).

[31] S.R. Amendolia et al. [NA7 Collaboration], Phys. Lett. B 178, 435 (1986).

[32] P. Maris and P.C. Tandy, Phys. Rev. C 62, 055204 (2000).

[33] J. Bijnens and P. Talavera, JHEP 0203, 046 (2002).

[34] M.D. Scadron, F. Kleefeld, G. Rupp, and E. van Beveren, Nucl. Phys. A 724, 391 (2003);

M.D. Scadron, G. Rupp, F. Kleefeld, and E. van Beveren, Phys. Rev. D 69014010 (2004).

[35] J. Lowe and M.D. Scadron, arXiv:hep-ph/0503100; hep-ph/0509333.

[36] F. Cardarelli, I.L. Grach, I.M. Narodetskii, E. Pace, G. Salme, and S. Simula, Phys. Rev. D 53, 6682 (1996).

[37] S.J. Brodsky and E. de Rafael, Phys. Rev. 168, 1620 (1968).

[38] M. Davier, S. Eidelman, A. Höcker, and Z. Zhang, Eur. Phys. J. C 31, 503 (2003).

[39] Jefferson Lab experiment E98-108, spokesperson P. Markowitz. 